\title{
AGENT-BASED SIMULATION OF HOUSEHOLD BANDWIDTH DEMAND
}

\section{Mathias Kern, Russell Ainslie, Doug Williams, Arnaud Jacquet, Nicola Wong}

Applied Research, BT

Adastral Park, Martlesham, Ipswich

mathias.kern@bt.com, russell.ainslie@bt.com,doug.williams@bt.com, arnaud.jacquet@bt.com, nicola.wong@bt.com

\begin{abstract}
In this paper we present a snapshot of our ongoing research into an agent-based model for simulating bandwidth demand at the household level. Motivated by Edholm's law on the exponential growth of internet traffic, our approach models households, persons and devices and their internet traffic generating interactions, and builds a picture of overall total and peak demand. Assumptions on the types, likelihoods and bandwidth requirements of various activities are key inputs to the simulation, and will allow us to test different hypotheses on future growth in the next phase.
\end{abstract}

Keywords: Agent-Based Simulation, Internet Traffic, Bandwidth, Household Model.

\section{INTRODUCTION}

The internet has seen phenomenal growth in users, traffic and applications over the last decades, and has now reached almost every aspect of human life. Figure 1 illustrates the exponential increase in global IP traffic since 1990 (Wikipedia: https://en.wikipedia.org/wiki/Internet_traffic, accessed 18 September 2019). This observation has led to the formulation of Edholm's law (Cherry 2004) which states that the required traffic data rates double every 1.5 years. However, internet traffic cannot continue on this growth trajectory forever, and a slow-down is expected at some point. This, of course, poses a significant challenge to businesses such as internet service providers, network infrastructure companies and network equipment producers. These businesses need to forecast likely future traffic and plan ahead and invest accordingly. In this paper, we introduce an agent-based model for the simulation of bandwidth demands at the domestic household level to draw conclusions about overall network bandwidth demands. We describe the makeup of the model, state key modelling assumptions, and discuss the current state of our on-going work and next steps.

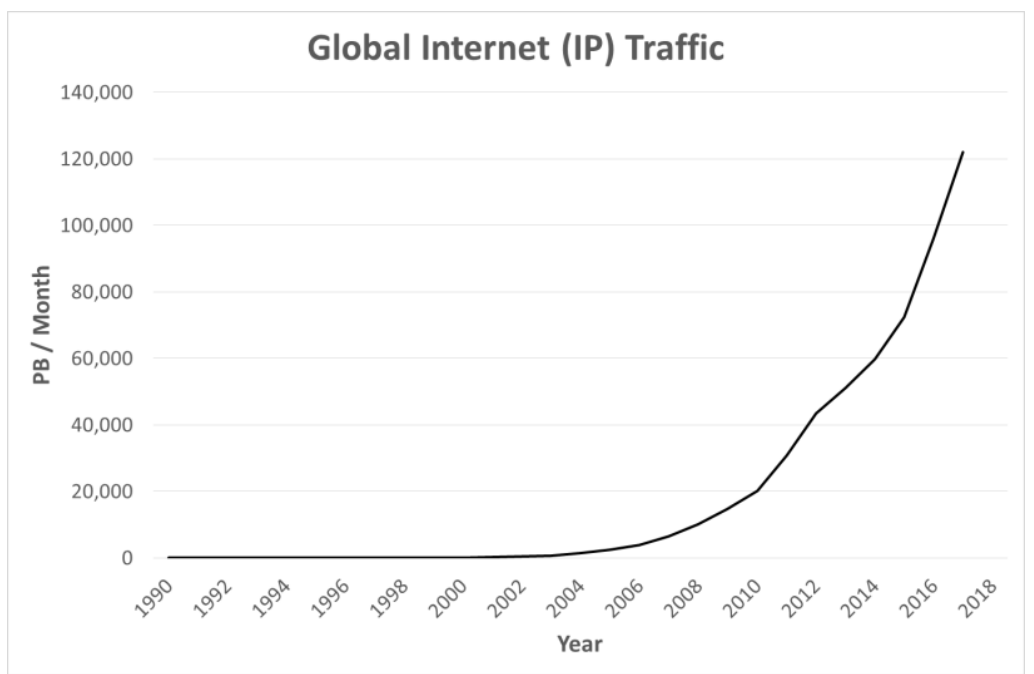

Figure 1 Global internet (IP) traffic by year in Petabyte / month 


\section{AGENT-BASED SIMULATION APPROACH}

In order to approximate and predict overall and peak internet traffic demand for domestic consumers, an agent-based approach, a method applied successfully to many scientific domains (Niazi and Hussain, 2011), has been chosen to simulate traffic patterns generated by residential households. We model households and people and devices within these households as agents, and simulate key interactions between them, specifically activities that generate internet traffic.

Key modelling considerations for our household bandwidth simulation include:

i. The types of people modelled such as 'young adult (18-34 years)' or 'retiree (65 years+)'.

ii. The types of devices simulated, e.g. 'personal device' or 'generic household device'.

iii. The types of households, for example. 'couple (45-54 years) with no children' or 'single adult (35-44 years) with two dependent children', which describe the make-up of the household.

iv. The types of network connection available to a household, for instance 'fibre broadband' or 'copper broadband'

v. The categories of activities associated with people such as 'watch', 'game', 'listen', 'communicate' and 'read', and specific activity types such as 'watch paid video-on-demand' or 'online gaming'

vi. The generic activity types associated with household devices such as 'background activity' or 'download of update'.

These choices form the foundations of the household bandwidth model, and allow us to specify different scenarios. Each simulation scenario is composed of a number of partial or mini-scenarios, and these can be easily re-used and re-combined to form new overall scenarios. Below is a list of some of the central configurations for constructing a scenario:

i. The number of households to simulate.

ii. The relative frequency of different household types which facilitates sampling of households.

iii. The relative frequency of the different network connection types for each household type.

iv. The internet traffic and speed characteristics associated with each network connection type.

v. Bandwidth requirements for each activity type, including start-up requirements (e.g. when starting to stream a movie online) and on-going requirements (e.g. to maintain the on-going streaming of a movie).

vi. The probabilities of starting a new activity by a person or device, by hour and minute of the day and by day of the week, individually for each person and device type.

The simulation steps through the $7 * 24 * 60=10,080$ minutes of a week minute by minute. In each step, we check for each person and device agent whether the agent has already an activity running, and/or whether a new activity should be started. For example, we allow person agents to have up to two activities live in parallel, e.g. watching an online video while also communicating at the same time, and decide stochastically whether and what activities to start. People and device agents send bandwidth requests to their respective household agents, which in turn might either fully satisfy or cap the actually allocated bandwidth, depending on whether the overall requested bandwidth is within or exceeds what the household's network connection can support. We collect activity and bandwidth data for each person, device and household agent for each minute cycle, and compute overall traffic, peak traffic and whether an agent's traffic is capped.

Three important aspects have to be combined to make the simulation truly functional. Firstly, the agent-based model itself, i.e. the 'mechanics' of the simulation, needs to be developed. Secondly, key scenario assumptions and inputs need to be compiled, e.g. information about the likelihoods and bandwidth requirements of different activities. And thirdly, actual bandwidth demand for a network of residential customers needs to be collected to facilitate the setup and tuning of the model and ensure that simulation results and real life observations match closely. 


\section{CURRENT STATE AND FUTURE WORK}

The work on an agent-based model for the simulation of household bandwidth demand is an active area of research and development, and this paper presents a current snapshot of our thinking and progress. Although we initially considered and experimented with implementing the simulation in AnyLogic (https://www.anylogic.com, accessed 11 September 2019), the current solution is implemented in Java to allow for more flexibility and easier development and sharing across teams. Furthermore, we have compiled a list of scenario configurations and assumptions, and they combine information from a diverse range of data sources, including the Office for National Statistics (ONS) for trends on households (ONS: Families and households, 2018) and the Office of Communications (Ofcom) for trends in internet usage (OFCOM: Internet use and attitudes, 2019). We have also sourced current actual aggregate network bandwidth usage statistics, which mirror the patterns previously published in (Lord et al, 2016) shown in figure 2. This has allowed us to run simulations for recent/current bandwidth use scenarios, and simulation results and actual observations match well. In summary, we have developed an agent-based household bandwidth simulation model and can closely replicate current real life observations with it.

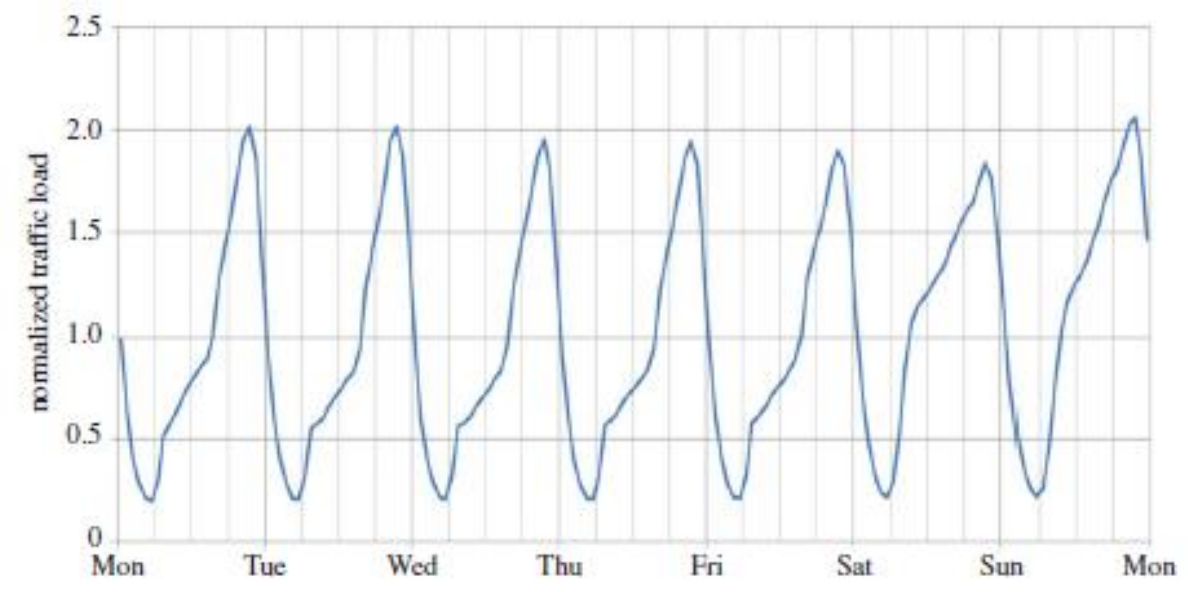

Figure 2 Typical distribution of traffic load across the week on a residential fibre network (1.0=average load) (Lord et al, 2016).

The next phase of our research and development work will address the following points:

- Support parallel running of simulations across a distributed computing infrastructure to facilitate large scale experiments.

- Build assumptions and data for future scenarios, including:

- Do we anticipate completely new, as of now unknown, activities, for instance in the augmented or virtual reality space, or in the internet of things domain?

- What are the possible bandwidth requirements for individual activities, e.g. for streaming online videos, over the next 10 to 20 years?

- Will certain activities become more or less frequent going forward, e.g. with the move from terrestrial TV to online streaming?

- Will we see a change in the online behaviour of certain age groups, i.e. person types, e.g. do we anticipate retirees to become more frequent internet users?

- Do we expect a social shift in the make-up of households over the next decades, e.g. do we foresee a higher proportion of single person households?

- Run simulations for likely / best-case / worst-case future scenarios.

- Ultimately, provide guidance for answering the central question: Do the simulation results indicate further exponential growth of network traffic, or do they point to a slowing of the growth rate?

- Overlay the household model with different network topology assumptions such as full fibre. 


\section{REFERENCES}

Cherry, Steven (2004). Edholm's law of bandwidth. IEEE Spectrum. 41(7): 58-60, doi:10.1109/MSPEC.2004.1309810.

Lord A., Soppera A., Jacquet A. (2016). The impact of capacity growth in national telecommunications networks. Philos Trans A Math Phys Eng Sci. 2016 Mar 6;374(2062).

Niazi, Muaz and Hussain, Amir (2011). Agent-based Computing from Multi-agent Systems to AgentBased Models: A Visual Survey. Scientometrics. 89 (2): 479-499.

OFCOM: Internet use and attitudes (2019). https://www.ofcom.org.uk/research-and-data/internetand-on-demand-research/internet-use-and-attitudes, accessed 18 September 2019.

ONS: Families and households: 2018. https://www.ons.gov.uk/peoplepopulationandcommunity/ birthsdeathsandmarriages/families/bulletins/familiesandhouseholds/2018, accessed 2 September 2019.

\section{AUTHOR BIOGRAPHIES}

MATHIAS KERN is a senior research manager in BT's Applied Research team, and his research interests focus on the application of AI, optimisation and simulation techniques to real-life resource management problems. He gained his $\mathrm{PhD}$ in Computer Science from the University of Essex researching population-based optimisation methods, and has been with BT since 2004 .

RUSSEL AINSLIE is a research specialist in AI and optimisation at BT Research whilst completing a PhD in large-scale optimisation problems at Robert Gordon University. Starting from a background of a BSc in Maths and Physics from Edinburgh and a BSc and MSc in Computer Science from RGU, Russell has developed specific expertise in workforce management.

DOUG WILLIAMS is a research manager in BT's Applied Research team, specialising in Multimedia Analytics.

ARNAUD JACQUET is a research manager in BT's Applied Research team, with a particular research interest in the area of quality of experience.

NICOLA WONG received a BSc (Hons) Computer Science from the University of East Anglia in 2016. She is currently a researcher in the Operational Transformation research theme at BT Applied Research. 